\title{
Wet rolling stones: Growth of a granular aggregate under flow
}

\author{
Pierre Jop ${ }^{1, *}$, Guillaume Saingier ${ }^{1,}$, and Alban Sauret ${ }^{2}$, \\ ${ }^{1}$ Surface du Verre et Interfaces, UMR 125, CNRS/Saint-Gobain, 39 quai Lucien Lefranc, 93303 Aubervilliers, France \\ ${ }^{2}$ Department of Mechanical Engineering, University of California, Santa Barbara, USA
}

\begin{abstract}
Wet granulation processes can be driven from low to high water content. In this study, we consider the model situation of the growth of a single wet aggregate rolling in a dry granular flow inside a rotating drum. We measure the time evolution of its diameter for different grains and liquids, as well as various rotation rates of the drum. Using X-ray tomography, we are able to characterize the internal structure of the granular aggregate at different times during the process. We show that the growth rate of the aggregate can be related to the transport of the liquid inside the granule and the capture of grains. We propose a model to rationalize the maximum size of the aggregate and its growth rate.
\end{abstract}

\section{Introduction}

Blending dry grains and liquid is a common step of many industrial processes. When the volume of liquid is small compared to the volume of grains, wet aggregates are created. The amount of liquid is typically measured through the volume ratio of liquid $W$. The value of $W$ delimitates different regimes of wet granular media: dry, pendular, funicular, capillary and suspensions [1]. In wet aggregates, the capillary forces hold the wet grains together, bringing strength to the object. Whereas many past studies have considered the static properties of wet granular material, the dynamical situation where grains and liquid are blend together remains more elusive.

The dynamical situation is nevertheless important in many practical applications. This phenomenon is, for instance, at the base of the wet granulation processes [2]. The wet aggregates interact with the surrounding medium and can collide, break, and exchange grains. In high shear granulators, the kinetic energy of the aggregates is essential. However, in industrial granulation dishes or in rotating drums, the granulation is done at low shear and allows the individual aggregates to preserve their shape and roundness. Depending on the liquid content of the aggregate, different mechanisms can occur. At very low water content, the grains at the surface of the aggregate can be eroded due to the friction of the granular media and to the impact of the dry grains [3]. At very large water content, the flowing dry grains can be captured if they touch a capillary meniscus [4].

To characterize how aggregates, initially saturated in liquid (i.e., in the capillary regime), evolve when flowing in a dense granular flow we consider here a model situation that allows us to obtain macroscopic and microcsopic properties of an individual aggregate during its evolution. In this study, we focus on an isolated wet aggregate during

*e-mail: pierre.jop@saint-gobain.com
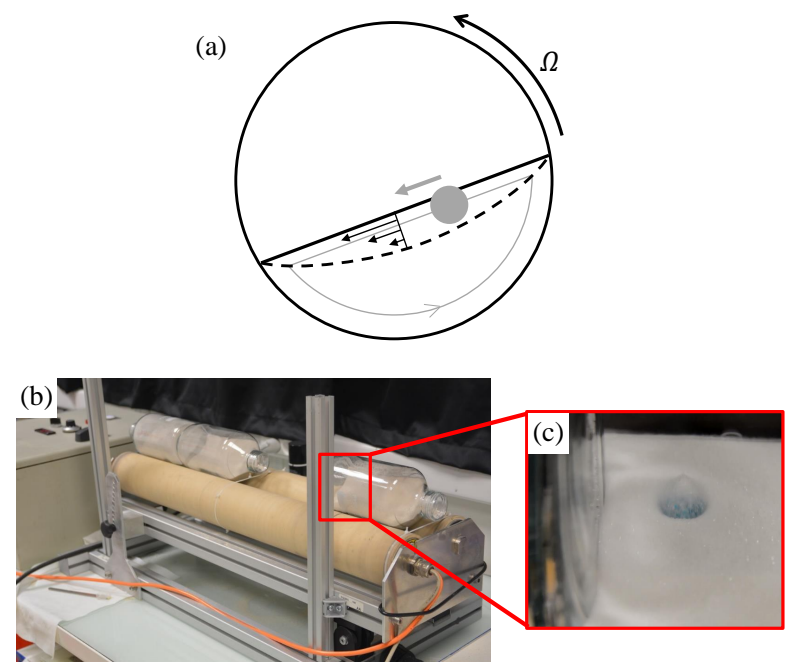

Figure 1. (a) Schematic of the aggregate rolling inside the surface flow of the granular media in a drum. Photography of (b) the experimental setup and (c) the initial aggregate consisting of dyed liquid and grains.

all of its evolution and describe the role of the kinematic and physical properties of the material on its growth. We characterize the time evolution of its mass and propose a model that rationalized our observations based on the transfer of liquid between domains of grains of different sizes.

\section{Experiments: growth dynamics of the wet aggregate in a rotating drum}

We use a rotating drum to measure the growth of a single aggregate in a gentle granular flow [Fig. 1(a)]. The drum is a long glass cylinder of diameter $10 \mathrm{~cm}$ and length $20 \mathrm{~cm}$ 


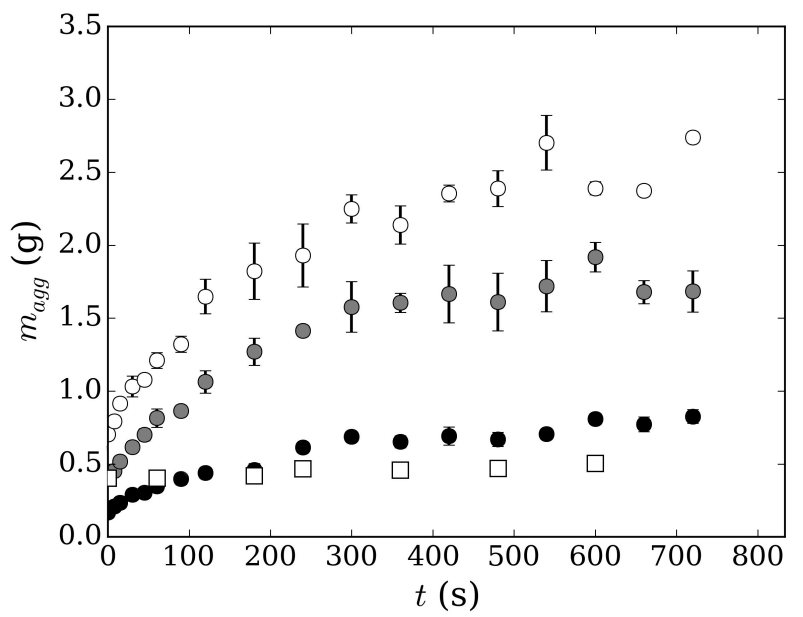

Figure 2. Time evolution of the mass of the aggregates of sand for increasing volumes of the initial droplet of $\mathrm{ZnI}_{2}$ solution (circles from dark to light) $20 \mu \mathrm{L}, 50 \mu \mathrm{L}, 100 \mu \mathrm{L}$ and comparison with a buried aggregate in a static media from a $20 \mu \mathrm{L}$ droplet (squares).

allowing the aggregate to travel in the flow without being subject to the effects of the side boundaries [Fig. 1(b)]. The cylinder is filled at $25 \%$ with sand of diameter $d_{g}=$ $60-300 \mu \mathrm{m}$ and can rotate from $0 \mathrm{rpm}$ to $40 \mathrm{rpm}$.

An aggregate is prepared by depositing a drop of aqueous solution of $\mathrm{ZnI}_{2}$ or glycerol on the surface of the sand before starting the rotation [Fig. 1(c)]. This method allows us to create an initial aggregate fully saturated with liquid. As shown in Fig. 1(a), the aggregate is then carried by the flow, partially sunk. It can easily be collected after several rotations to characterize its properties, such as its mass. Instead of measuring the diameter, we weighted the aggregates to get rid of the aggregate shape that may depart strongly from a regular sphere.

Fig. 2 shows the temporal evolution of the mass of the aggregates for different initial volumes of a water solution of $\mathrm{ZnI}_{2}$. The mass increases with time $t$, and the growth rate slows down until reaching a saturation. There is a strong increase in the mass of the aggregate as a function of time as it is multiplied by about 3 in 5 minutes, before slowing down and reaching an approximately constant value of 4 times the initial mass of the aggregate.

The increase in mass results from the capture of dry grains by the air/liquid menisci at the surface of the aggregate. We do not usually observe clusters of grains detaching from the aggregates. However, at the end of the experiment, a large aggregate can break into several parts. The aggregate grows by gluing new sand grains on its surface without exchange with the surrounding media in contrast with a recent numerical study [5]. To test if the spontaneous capillary spreading of the liquid toward new dry grains plays a key role, we also report the time evolution of the mass of the same initial aggregate buried in the sand but without flow. We observe that the mass grows by less than $25 \%$. Therefore the capture mechanism of dry grains requires a granular flow and is reminiscent of the growth
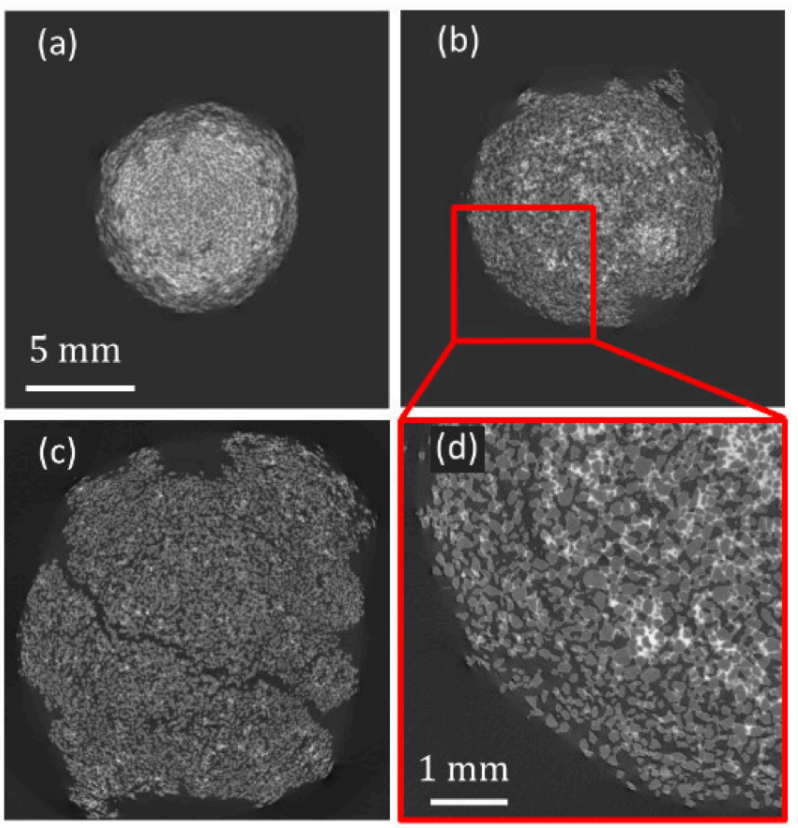

Figure 3. Section of X-ray tomography of aggregates of sand grains at (a) $t=0 \mathrm{~s}$, (b) $t=100, \mathrm{~s}$, (c) $t=360, \mathrm{~s}$ and (d) magnification showing the spreading of the liquid.

of wet granular towers [4]. Moreover, as we increase the initial volume of liquid, the mass ratio between the initial and the final aggregate is of the order of 4 in all the experiments performed here. This behavior suggests that the evolution of the aggregate can be rescaled by the initial volume of liquid, and the transport of the liquid may be the key parameter.

\section{Microstructure of the aggregate during its evolution}

Our initial observations suggest that the granular flow around the aggregate is responsible for the growth of the isolated aggregate. Such an increase in mass requires liquid transport within the granular matrix to allow the capture of new sand grains. We collect some aggregates at different times and characterize their structure through Xray tomography, as illustrated in Fig. 3(a)-(d), which report a meridional section of the aggregates. The solution of $\mathrm{ZnI}_{2}$ provides a good contrast to observe the liquid distribution in the aggregate. While the pores at the center of the initial aggregate are fully saturated, the local amount of liquid decreases in the outer part. We observe a shell of captured sand grains that are smaller than the initial ones. Moreover, only a fraction of those pores is filled with the liquid. This behavior likely occurs because of the larger probability for the smaller grains, continuously brought to the aggregate by the flow, to get captured. The pore size in the shell is then smaller, and the capillary pressure drops when the liquid starts invading them. As a result, the liquid is pumped out from the core consisting of large pores until it is empty. This hypothesis also explains why the aggregate at rest does not grow: the size distribution of the 


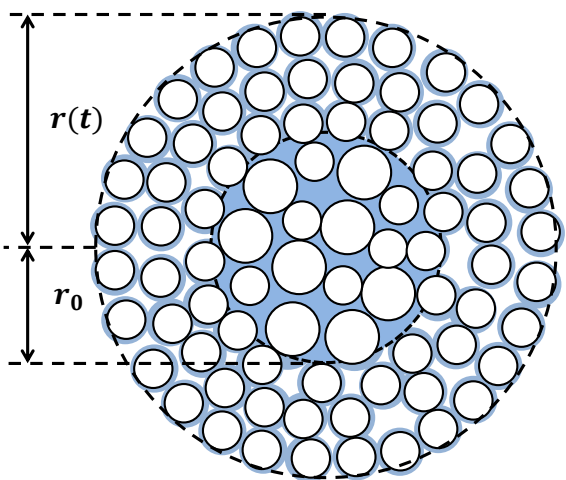

Figure 4. Schematic of the transport of liquid from the saturated core of the aggregate to the periphery. The liquid moves along the grains, exploring a secondary porosity consisting in smaller pores than in the initial porosity of the aggregate.

dry grains in contact with the wet aggregate is the same as the ones inside.

\section{Model: Growth dynamics through the transport of liquid}

Based on this physical argument and using geometrical assumptions, we can propose a model for the growth rate of this aggregate. We first assume that the core of the aggregate is composed of large grains (Fig. 4) with a shell consisting of smaller grains. Secondly, the volume fraction of the pores is assumed to be constant everywhere. Thirdly, the growth stops when the liquid volume fraction drops below a value that does not allow a continuous liquid path. The value of this percolation threshold was estimated by Scheel et al. [6] to be of the order of $W=0.05$, while the initial value for this sand is $W_{0}=(1-\phi) / \phi \simeq 0.43$. Finally, the transport of the liquid is governed by a Darcy flow whose pressure gradient is given by the capillary pressure inside the two types of pores.

More precisely, we assimilate the saturated core of the aggregate to a liquid reservoir, feeding a three-dimensional imbibition in a granular material and thus the growth of the aggregate, through a network of channels, of permeability $k_{\text {eff }}$. We develop here a radial imbibition model from an extended point source. We note $r_{0}$ the initial radius of the saturated aggregate and $r(t)$ the radius of the aggregate at time $t$, assimilated to the position of the impregnation front [Fig. 4]. The liquid is mobilized by the capillary depression resulting from the accretion of small grains on the surface of the aggregate, which creates pores of radius $r_{p}$, and pumps the liquid from the core to the surface. It is further assumed that the growth is only radial, and we note $q(t)$ the flow rate of liquid at the front. The Darcy equation relates the pressure gradient to the radial flow velocity and can be written as a function of the flow rate $q(t)$ :

$$
-\frac{\mathrm{d} p}{\mathrm{~d} r}=\frac{\eta q(t)}{4 \pi r^{2} k_{\mathrm{eff}}}
$$

where $\eta$ is the viscosity of the liquid. The pressure at the boundary of the saturated core is related to the local curva-

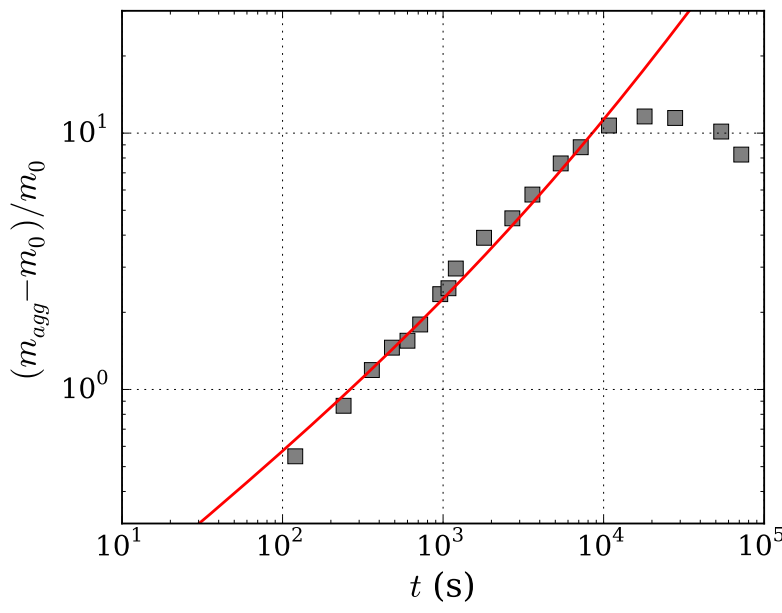

Figure 5. Time evolution of the mass of the glycerol/sand aggregate (square) and the prediction of the model (line).

ture of the menisci and is $-2 \gamma / R_{c}$, where $\gamma$ is the surface tension of the liquid. The pressure at the front is $-p_{c}^{p}=$ $-2 \gamma \cos \theta / r_{p}$. By substituting $q(t)$ by its expression as a function of the local radial velocity $q(t)=4 \pi(1-\phi) r^{2} u_{r}$, we obtain

$$
r^{2} \frac{\mathrm{d} r}{\mathrm{~d} t}\left(\frac{1}{r}-\frac{1}{r_{0}}\right)=\frac{k_{\mathrm{eff}} \Delta p_{c}}{(1-\phi) \eta}
$$

Integrating this relation allows to obtain an implicit expression of the imbibition radius $r(t)$ which then leads to the mass of the aggregate over time and finally to the normalized mass variation as a function of the aggregate radii such as

$$
\frac{m_{\mathrm{agg}}-m_{0}}{m_{0}}=\frac{r^{3}-r_{0}^{3}}{r_{0}^{3}}\left(\frac{\phi \rho_{g}}{\phi \rho_{g}+(1-\phi) \rho}\right) \propto \frac{r^{3}-r_{0}^{3}}{r_{0}^{3}}
$$

We validate this model using glycerol, which makes stronger aggregates and which wets well the sand grains. It should be noted that a rigorous comparison is tricky because the capillary pressure difference and permeability $k_{\text {eff }}$ can only be estimated and not measured or calculated directly. However, these two parameters do not affect the shape of the power law in logarithmic plot, but only shift it with time by modifying the prefactor. By taking $r_{0}=3 \mathrm{~mm}$ in the model, according to the experimental observations for $V=50 \mu \mathrm{L}$, the theoretical prediction corresponds well to the experimental measurements as shown in Fig. 5. The agreement between the theoretical model and the experimental data seems to indicate that the growth dynamics of an isolated aggregate in a dense granular flow results from the radial capillary imbibition of an unsaturated granular material from a large spherical source of finite volume. In this case, the growth of the aggregate is limited by the viscous displacement of the liquid in the granular network and not by the capture rate of the grains. However, the movement of the grains surrounding the aggregate is essential to generate the pressure gradients necessary to set the fluid in motion, promoting the selection and sticking of small grains at the periphery of the aggregate. 


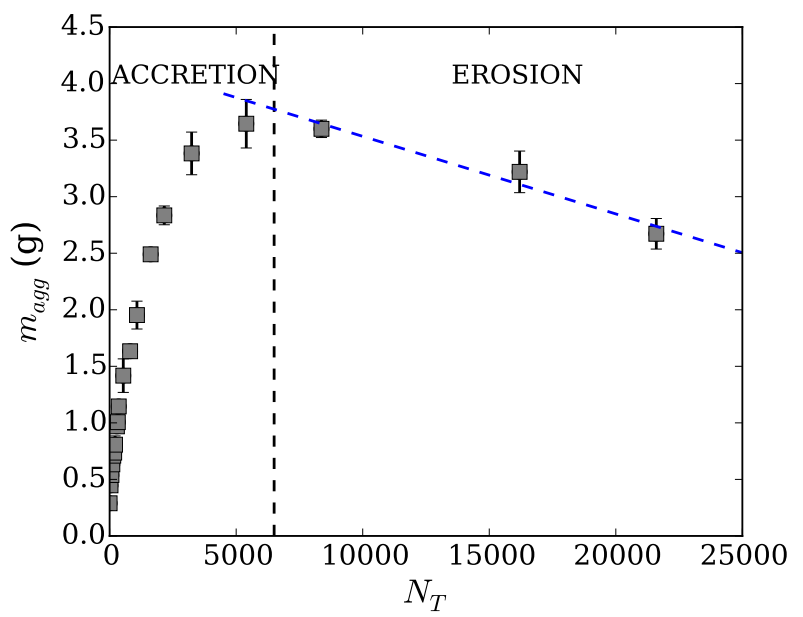

Figure 6. Illustration of the succession of the accretion and then erosion phases during the evolution of an aggregate of sand and glycerol in a granular flow.

\section{Beyond the growth: erosion of the aggregate}

After the growth phase, the expansion of the aggregate begins to slow down, and the previous description, assuming an infinite reservoir of liquid in the saturated core, is no longer relevant. Visualizations by X-Ray tomography show that the core, initially saturated with liquid, has largely emptied and that the liquid is distributed throughout the aggregate in the form of more or less connected capillary bridges. The volume fraction of liquid $\mathrm{W}$ in the aggregate then becomes small, of the order of $W \sim 0.05$. Below this threshold, liquid transfers become very difficult [6], and the growth stops. Besides, the aggregate is susceptible to be eroded by the flow of grains. Fig. 6 shows the evolution of the mass of the aggregate as a function of time for a mixture of sand and glycerol. The erosion rate shown here is quite low due to the low inertia of the dry grains interacting with the aggregate and the high viscosity of the glycerol. This type of dynamics by accretion/erosion has already been observed in experiments with static aggregates in a rotating drum [3].

\section{Conclusion}

In this study, we characterized the growth of an aggregate, initially saturated in liquid, in a gentle granular flow. Using rotating drum experiments, we demonstrated the existence of a growth phase during which the aggregate capture grains by accretion until it reaches a critical size and enters an erosion phase during which the surrounding flow erodes the aggregate. During the growth phase, we have shown that a shell of smaller grains develops around the core made of larger grains and drives the liquid outside under the effect of capillary pressure. Using a radial capillary imbibition model from a finite source, we have determined a theoretical solution that correctly predicts the scaling law describing the experimentally measured mass increase. These different results are of interest for low shear rate granulation processes, traditionally used to produce large aggregates from grains of about 100 micrometers. A better control of the growth of aggregates and their structure could allow to develop more controlled aggregates or with more complex compositions.

\section{References}

[1] N. Mitarai, F. Nori, Adv. Phys. 55, 1-45 (2006)

[2] S.M. Iveson, J.D. Litster, K. Hapgood, B.J. Ennis, Powder Technol. 117, 3-39 (2001)

[3] G. Lefebvre, P. Jop, Phys. Rev. E 88, 03220 (2013)

[4] G. Saingier, A. Sauret, P. Jop, Phys. Rev. Lett. 118, 208001 (2017)

[5] T.T. Vo, S. Nezamabadi, P. Mutabaruka, J.Y. Delenne, E. Izard, R. Pellenq, F. Radjai, Eur. Phys. J. E 42, 127 (2019)

[6] M. Scheel, R. Seemann, M.D. Brinkmann, M. Di Michiel, A. Sheppard, B. Breidenbach, S. Herminghaus, Nat.Mat. 7, 189-193 (2008) 